\title{
Correction to: Discrimination threshold for haptic volume perception of fingers and phalanges
}

\author{
Zhilin Zhang $^{1} \cdot$ Chunlin $\mathrm{Li}^{2} \cdot$ Jian Zhang ${ }^{1} \cdot$ Qiang Huang ${ }^{1,3} \cdot$ Ritsu Go ${ }^{1,4} \cdot$ Tianyi Yan ${ }^{5} \cdot$ Jinglong Wu $\mathbf{W}^{1,4}$ \\ Published online: 24 November 2020 \\ (C) The Psychonomic Society, Inc. 2020
}

\section{Correction to: Atten Percept Psychophys. https://doi.org/10.3758/s13414-017-1453-z}

An incorrect symbol appeared in the Fig. 3 caption of the published article. The correct symbol for the caption appears below along with the correct artwork.

The online version of the original article can be found at https://doi.org/ $10.3758 / \mathrm{s} 13414-017-1453-\mathrm{z}$

Tianyi Yan

yantianyi@bit.edu.cn

1 Intelligent Robotics Institute, School of Mechatronical Engineering, Beijing Institute of Technology, Beijing 100081, China

2 School of Biomedical Engineering, Capital Medical University, Beijing 100069, China

3 International Joint Research Laboratory of Biomimetic Robots and Systems, Ministry of Education, Beijing 100081, China

4 Key Laboratory of Biomimetic Robots and Systems, Ministry of Education, Beijing 100081, China

5 School of Life Science, Beijing Institute of Technology, Beijing 100081, China 

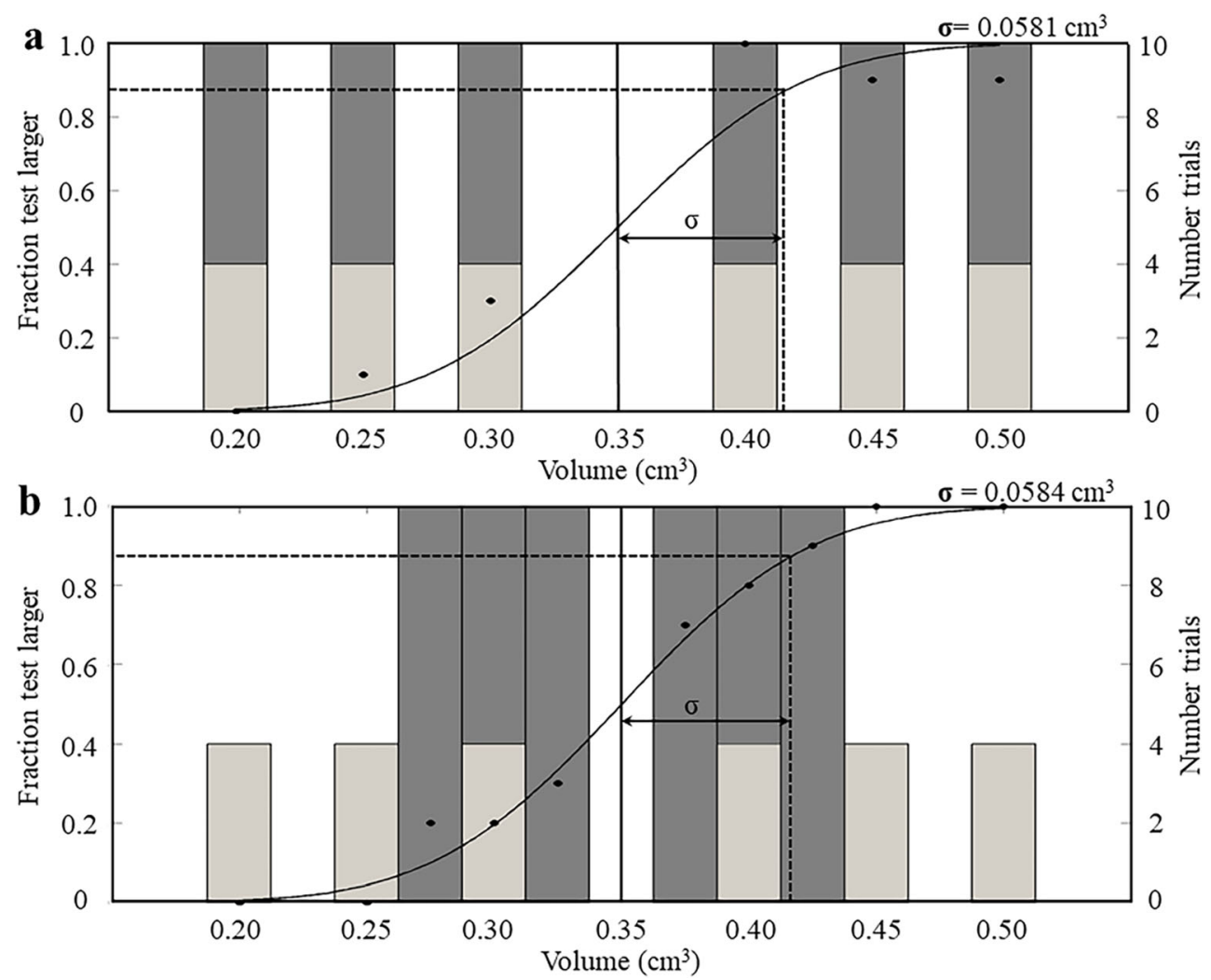

Fig. 3 a, $\mathbf{b}$ The value of this discrimination threshold, $\sigma$, is shown in the upper right corner of the figure

Publisher's note Springer Nature remains neutral with regard to jurisdictional claims in published maps and institutional affiliations. 